\title{
Benefits of radial distortion correction in arthroscopic surgery: a first experimental study on a knee model
}

\author{
Rui M. Duarte ${ }^{1,2,3 *}$ \\ Nuno V. Ferreira ${ }^{1,2,3}$ \\ Armando M. Oliveira ${ }^{7}$ \\ Fernando P. Fonseca ${ }^{5,6}$ \\ Manuel Vieira-Silva ${ }^{1,2,3}$ \\ Jorge Correia-Pinto ${ }^{1,2,4}$ \\ ${ }^{1}$ Life and Health Sciences Research \\ Institute (ICVS), School of Health \\ Sciences, University of Minho \\ ${ }^{2}$ ICVS/3B's PT Government Associate \\ Laboratory \\ ${ }^{3}$ Orthopedic Surgery Department, \\ Hospital de Braga \\ ${ }^{4}$ Pediatric Surgery Department, \\ Hospital de Braga \\ ${ }^{5}$ Orthopedic Surgery Department, \\ Centro Hospitalar da Universidade de \\ Coimbra \\ ${ }^{6}$ Medical School, University of \\ Coimbra \\ ${ }^{7}$ University of Coimbra, Institute of \\ Cognitive Psychology - Faculty of \\ Psychology and Educational Sciences \\ *Correspondence to: Rui M. Duarte, \\ Life and Health Sciences Research \\ Institute (ICVS), School of Health \\ Sciences, University of Minho. \\ Email: ruimfduarte@ecsaude. \\ uminho.pt
}

Accepted: 28 July 2014

\begin{abstract}
Background Lens probes used in arthroscopy typically have a small diameter and wide field-of-view. This introduces strong radial distortion (RD) into the image, ultimately affecting the surgeon's hand-eye coordination. This study evaluates potential benefits of using distortion-free images in arthroscopic surgery.
\end{abstract}

Methods Distortion-free images were obtained using RDFixer ${ }^{\mathrm{TM}}$ software (Perceive3D, SA) to remove RD in the input video stream. Twelve orthopedic residents performed an arthroscopic task (loose body removal) in a dry-knee model using video with and without distortion. Residents were questioned about image quality, and surgical performance was rated using an adapted Global Rating Scale.

Results A statistically significant improvement of all parameters was observed with distortion-free images. Residents perceived distortion-free images as providing a wider field-of-view and a better notion of relative depth and distance.

Conclusion $\mathrm{RD}$ correction improved the surgical performance of residents, potentially decreasing their learning curve. Future work will study whether the benefits are observable in experienced surgeons. Copyright (C) 2014 John Wiley \& Sons, Ltd.

Keywords radial distortion correction; arthroscopy; depth perception; field of view

\section{Introduction}

In recent years, minimally invasive surgery (MIS) has become increasingly popular in many medical fields and is used not only for diagnosis but also in surgical treatments. MIS requires the use of a camera with a miniature lens that is inserted into the human body through a small incision or a natural orifice, as in the recently developed natural orifice translumenal endoscopic surgery (NOTES) technique (1-3). The images acquired by this camera allow the surgeon to observe the interior of anatomical cavities and control the action of instruments that are manipulated from the outside. Compared with the equivalent open surgery, MIS has the advantage of minimizing trauma, which results in lower complications rates (4), faster patient recovery (5), and shorter hospital stays (6). However, minimally invasive procedures, also referred to as keyhole surgeries, have the disadvantage of being technically 
more demanding (7). The use of long surgical instruments with reduced haptic feedback and limited degrees of freedom, as well as the fact that the entire procedure is executed using endoscopic video as the only guidance, make hand-eye coordination difficult to accomplish (8).

It is known that the correct perception of anatomical structures and relative depths may reduce complications and contribute to the clinical success of surgeries (9). Way et al., in a performance analysis of 252 laparoscopic bile duct injuries, identified inaccurate visual perception as the cause of $97 \%$ of all such error cases (10). Therefore, it has often been concluded that MIS requires a greater learning curve than conventional surgery (11).

Visual perception in general, and depth perception in particular, has always been an issue in MIS. Given that procedures are executed using endoscopic images as the only guidance, the surgeon has to undergo a long training period to learn how to infer 3D anatomical structures from 2D video and ultimately master the surgical techniques (11). In addition, several studies show that inaccurate depth perception results in orientation difficulties during the endoscopic procedure, ultimately hindering a precise execution of the task (12), increasing operating time and eventually also leading to surgical complications and clinical consequences for the patient.

Intensive research in stereo endoscopy has been conducted in the attempt to overcome depth perception issues. This technology, scientifically known as stereoscopy but commonly dubbed 3D visualization, typically requires two independent optic channels inside the endoscopic tube to provide a distinct view for each eye. These views are combined in the brain to give the perception of 3D depth. Despite the proven benefits of 3D endoscopy in surgical performance (13-17), the technology still presents some drawbacks: (i) it requires replacing the existing 2D endoscopic equipment with a new system; and (ii) the switch from 2D visualization to 3D endoscopy is a major shift in paradigm, whose operationalization by the surgeon requires the use of glasses, which often leads to fatigue and dizziness (18-22).

Small diameter lenses with a wide field of view, such as those used in orthopedic surgery, pediatric surgery, urology and neurosurgery (23), introduce an optical aberration in the images known as radial distortion (RD) or the fish-eye effect. Radial distortion is a nonlinear geometric deformation of the image, with the points being moved radially towards the center. This strong distortion severely affects the perception of relative size and depth (24). A study of the open-biopsy forceps measurement technique compared the error frequency when using original vs. corrected endoscopic images (where the radial distortion was removed). The mean error of the forceps technique in vivo using the original image was $26.5 \%+/-5.7 \%$ (under-estimation of size), which improved significantly to an error of $2.8 \%+/-3.2 \%$ with the distortion correction (25). The RD effect is illustrated in Figure 1, where the left side shows an image of a checkerboard acquired with a standard $4 \mathrm{~mm}$ arthroscope. The straight lines appear curved, giving the impression that the observed surface has a spherical shape. The right side of Figure 1 represents the same frame after distortion correction, using image-processing techniques through which the plane surface can be clearly perceived. Thus, given that $\mathrm{RD}$ clearly hinders depth perception and can potentially change the morphology of anatomical landmarks (9), it is reasonable to question the extent to which this optical aberration can affect surgical performance.

Previous studies have addressed the issue of RD in medical endoscopy and reported that distortion correction improved depth perception, in both 2D endoscopy and the new 3D endoscopy (26), while reducing the frequency of errors in activities of diagnosis $(9,12)$. However, to the best of our knowledge, no study has been conducted to assess the effect of $\mathrm{RD}$ in surgical performance, which means that this work is the first of its type. This lack of studies can be explained by the inexistence of a
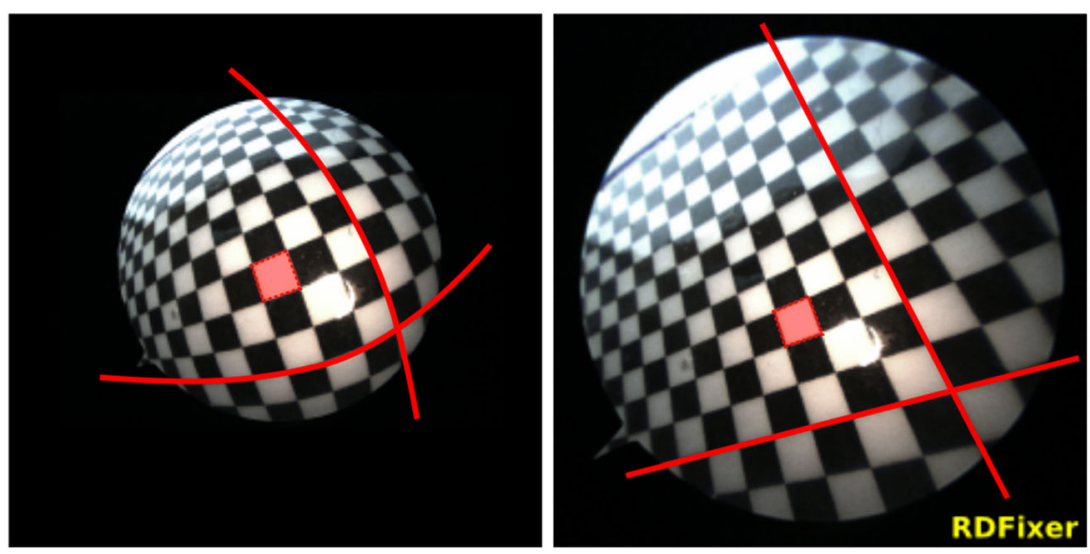

Figure 1. The effects of radial distortion: the left chessboard pattern (image with distortion) appears to have a spherical shape 
technology that is able to remove the radial distortion in real time. In this article, we use a prototype of the RDFixer $^{\mathrm{TM}}$ that has been kindly provided by Perceive3D, S.A. to conduct the experiments. RDFixer ${ }^{\mathrm{TM}}$ is a softwarebased system that calibrates the endoscopic camera and performs on-the-fly corrections of image radial distortion (27). It can be used with any type of medical endoscopic technology, including oblique-viewing endoscopes and high-definition (HD) image acquisition. This correction aims to enhance depth perception and improve visualization during endoscopic procedures, ultimately improving surgical performance and reducing the technical learning curve. To test the system, we evaluated the performance of inexperienced orthopedic surgeons in performing common arthroscopic procedures with and without radial distortion correction.

\section{Materials and methods}

\section{Studied population}

A population of 12 arthroscopic surgeons under training, with different levels of expertise, were recruited to take a blind test consisting of the execution of a common

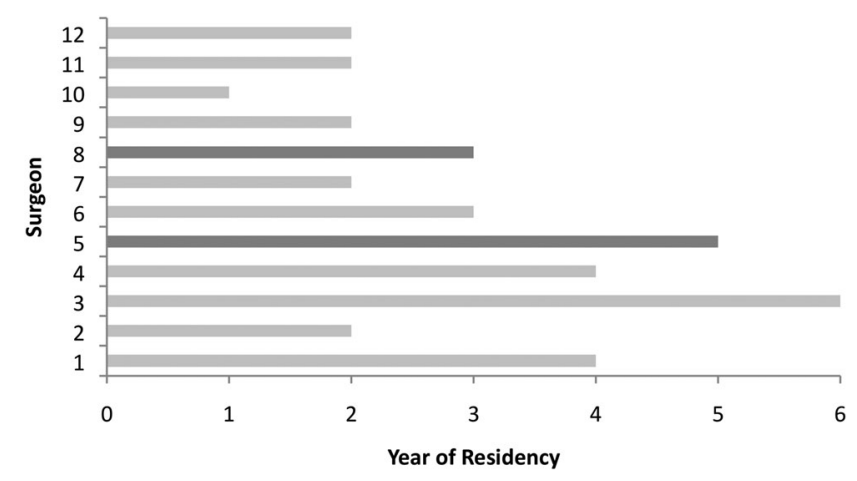

Figure 2. Random distribution of surgeons according to year of residency and level of arthroscopic experience: light grey $<5$ arthroscopic procedures, dark grey 5-20 arthroscopic procedures arthroscopic task in a dry knee model. During this blind test, the surgeon had no information regarding the existence of any manipulation of the image and/or the visualization system used. Figure 2 presents the level of arthroscopic experience of the orthopedic surgeons enrolled in this study.

\section{The RDFixer ${ }^{\mathrm{TM}}$ system}

The RDFixer ${ }^{\mathrm{TM}}$ system is a Plug\&Play device that is connected between the endoscopy tower and the medical display to correct the image radial distortion in real time (Figure 3). The system is composed of commercial offthe-shelf (COTS) hardware, and the image correction is performed by the software. RDFixer ${ }^{\mathrm{TM}}$ can be used with any endoscopy tower that is available in the market, and it can potentially be used in a wide range of clinical fields. Figure 4 shows the result of radial distortion correction of an arthroscopic image obtained within a knee model, where the difference in depth perception and the morphology of the anatomical structures can be perceived.

The RDFixer $^{\mathrm{TM}}$ system relies on an initial calibration procedure that consists of determining the optical parameters of the endoscopy system to correctly remove radial distortion. This endoscope calibration is performed at the beginning of each procedure and requires the surgeon to take a picture of a calibration target (Figure 1). The camera parameters are then automatically computed in a few seconds without the need for any user intervention (27).

In terms of usability, the RDFixer ${ }^{\mathrm{TM}}$ system presents two decisive factors for enabling visualization improvements in MIS using a software-based approach:

1. Given that the surgeon often exchanges lenses in the operating room (OR), the manufacturer cannot calibrate the system in advance. Actual solutions for calibrating a camera require the acquisition of multiple images of a known pattern and substantial user intervention (this is usually a procedure performed in research laboratories), which makes it impractical to

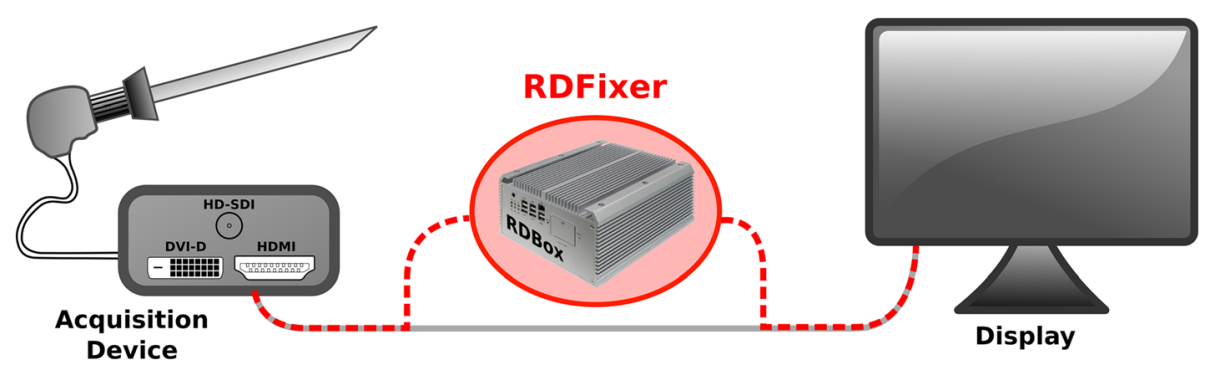

Figure 3. The RDFixer ${ }^{\mathrm{TM}}$ system is connected between the endoscopy camera control unit and the medical display, correcting the radial distortion in real time 


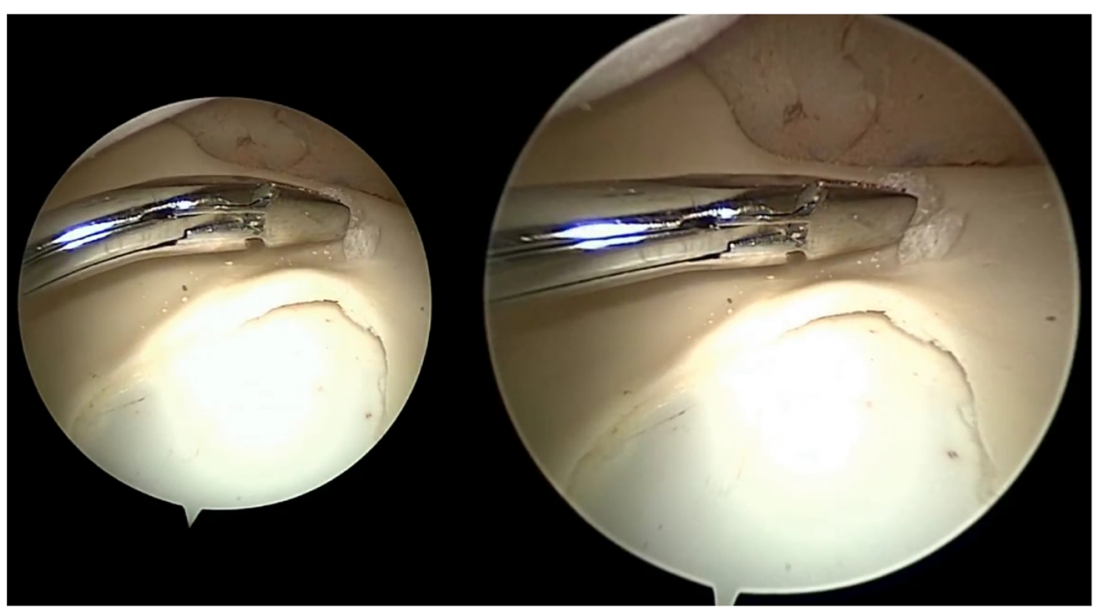

Figure 4. Radial distortion correction of an arthroscopic image obtained within a knee model

use in the OR. The RDFixer ${ }^{\mathrm{TM}}$ solution is unique because it makes it possible to calibrate the camera using a single frame acquired from an arbitrary pose. The calibration procedure is completely automatic and can be quickly performed in the OR before the clinical procedure starts, without interfering with the normal clinical routine (27).

2. The image warping necessary for correcting the radial distortion is carried in the heterogeneous architecture of the RDFixer ${ }^{\mathrm{TM}}$ system, using GPGPU techniques (GeneralPurpose Computing on Graphics Processing Units) (27). This enables the system to process $\mathrm{HD}$ video streams at high frame rates (such as 1080p@60Hz)

\section{Experimental setup}

An arthroscopy knee model (1401 dry application, Sawbones ${ }^{\circledR}$, Malmo, Sweden) was used to perform a common orthopedic arthroscopic procedure: loose body removal from the lateral compartment. For the experimental setup, two endoscopy towers were prepared and (222020-20, KARL STORZ GmbH \& Co. KG, Germany) equipped with two identical 30 degree cut arthroscopic lenses. Tower A was prepared to display the original view, and tower $\mathrm{B}$ had the RDFixer ${ }^{\mathrm{TM}}$ system installed, as shown in Figure 5. The resolution of both images, with or without correction, was $1920 \times 1080$ pixels displayed at $60 \mathrm{~Hz}$.

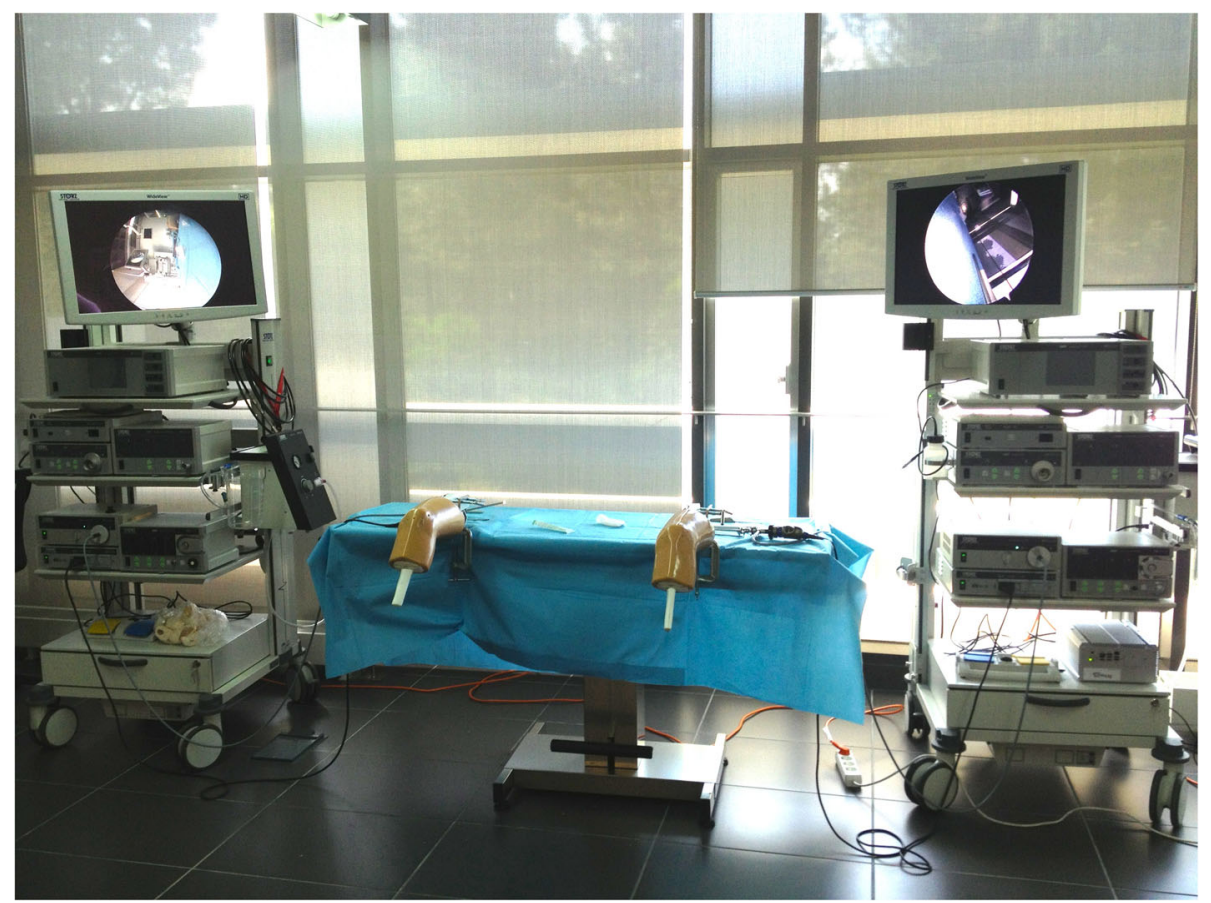

Figure 5. Experimental setup: photograph of endoscopic towers and knee models 
The tests were conducted in a dedicated room. Every procedure was supervised and scored by two experienced orthopedic surgeons (supervisor). From the surgeons' and supervisors' point of view, the two arthroscopy setups were completely identical. Residents were asked to enter the dedicated room individually and in a random order, and they were invited to perform the removal of a loose body using each of the visualization systems in a sequential manner. The balance in the order of using tower A or tower B was guaranteed following a subject randomization system. After each procedure, both supervisors scored the clinical performance of the surgeon, who then completed a survey about the visualization experience. The time occupied with the survey was also useful as a washout period between the two procedures.

\section{Evaluation form}

In this study, the Global Rating Scale (GRS) was used for evaluation of performance (Insel et al. (28)). This scale measures how well tasks are completed on a 1 to 5 point Likert-type scale. In this study, the scale was adapted for the model used, including four performance parameters:

i. instrument maneuver (IM), where 1 is given when the surgeon 'repeatedly makes tentative or awkward movements with instruments' and 5 when the surgeon makes 'fluid moves with instruments and no awkwardness';

ii. depth perception (DP), where 1 is given when the surgeon 'constantly overshoots target, slow to correct' and 5 when the surgeon 'accurately directs instruments in the correct plane to target';

iii. efficiency (E), where 1 is given when the surgeon performs 'many unnecessary, inefficient movements, constantly changing focus or persisting without progress' and 5 is given when the surgeon is 'confident, with clear economy of movement and maximum efficiency';

iv. quality of procedures (Q), where 1 is given when the quality is 'very poor' and 5 is given when the quality is 'clearly superior'.

\section{Subjective survey}

Each surgeon that performed the procedure answered a survey where three dichotomous questions were addressed: (i) What was your perception about the use of a correction method? (ii) Did you perceive the existence of a wider field of view? (iii) What was your perception about the existence of improved depth of the field of view?

\section{Data analysis}

Demographic data including age, gender, dominant hand and previous experience in arthroscopy were collected beforehand and analyzed. The IBM SPSS Statistics (version 20) software was used for data analysis. The results of the objective analysis obtained through the evaluation form were analyzed using the Marginal Homogeneity Test, while the results obtained from the subjective survey were analyzed using the McNemar Test. The unilateral significance level was used and obtained through exact methods, and a 0.05 criteria value was established.

\section{Results}

The population of twelve arthroscopic surgeons included ten males and two females, with ages ranging from 25 to 34 years (average age 29.4). All surgeons were righthand dominant. Regarding year of residency, $50 \%$ were in the first or second year, 33\% in the third and fourth, and $17 \%$ in the last 2 years.

Despite this distribution, only two members of the population had some previous experience in arthroscopic surgery (five or more procedures).

Objective analysis of the surgeons' performance was obtained through the evaluation form, where the response scale from 1 to 5 was taken as ordinal. Because comparisons involved the same surgeons, the Marginal Homogeneity Test was used. Because the superiority of the results in the condition with the corrected image (hypothesis-driven) was to be tested, the significance level used was unilateral. Because the number of subjects was large enough, the level of significance was obtained using exact methods (not asymptotic). Figure 6 indicates a statistically significant improvement in all four evaluated parameters when RDFixer ${ }^{\mathrm{TM}}$ radial distortion correction was used during the arthroscopic procedure: instrument maneuver $(P=0.008)$, depth perception $(P=0.001)$, and quality of procedures $(P=0.003)$, with a special emphasis on improved procedure efficiency $(P<0.001)$.

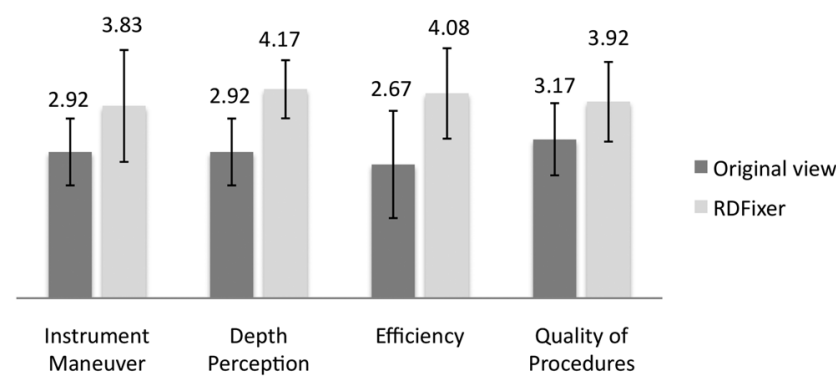

Figure 6. Objective analysis of surgeons' performance obtained through the adapted GRS scoring tool 
Conversely, the responses to the subjective survey were dichotomous (yes/no), and comparisons involved the same surgeon (paired). Therefore, a McNemar test was performed to assess statistical significance. The significance level was unilateral for the reasons indicated above and calculated by exact methods.

As observed in Figure 7 and Table 1, despite the surgeons' lack of perception about the use of a correction method to manipulate the image $(P=0.188)$, it was clear that there was a perception of a wider field of view when using RDFixer $^{\mathrm{TM}}$ radial distortion correction $(P=0.031)$ and improved depth perception during the procedure $(P=0.035)$.

\section{Discussion}

Radial distortion is a well-known deformation of the endoscopic image that severely affects the perception of relative size and depth. The RDFixer ${ }^{\mathrm{TM}}$ software was used in this study to remove $\mathrm{RD}$ during arthroscopic procedures. This study was conducted with a small population

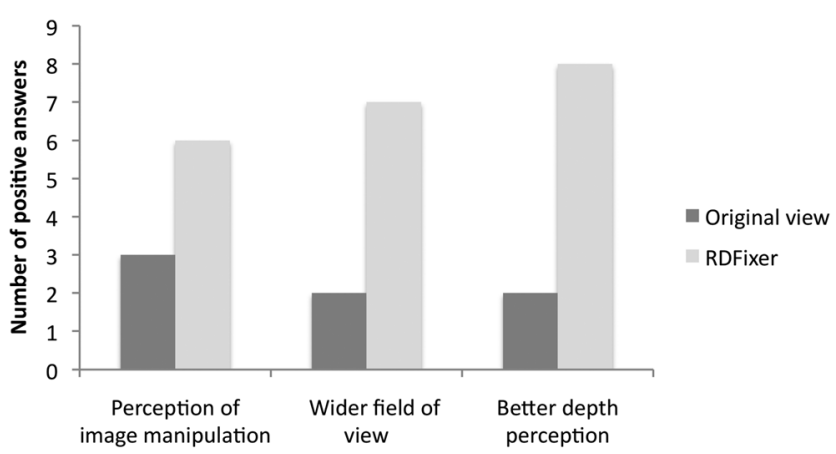

Figure 7. Subjective analysis of surgeons' perception obtained through a survey of inexperienced orthopedic residents given that, at this stage, surgeons have less capacity to overcome the challenges and limitations given by the distorted image.

An adapted GRS score was used as a well-established scale to evaluate arthroscopic skills during training periods (28). The adaptation of the scale consisted of the exclusion of parameters that were not relevant or applicable to the study model. Upon data analysis, it was observed that RD correction seems to have had an effect on the majority of the surgeons' performances, given that a significant improvement in their tasks was obtained with this image correction. Given that only two of the surgeons were slightly more experienced ('Level of Expertise' - Table 1), it was not possible to determine the influence of this variable on the outcomes. No significant correlations (Spearman Test) were obtained between the years of residency and improved ratings.

Answers to the subjective survey provided valuable outcomes. Most surgeons did not perceive that there was any image manipulation, compared with their previous experience; this was a positive observation, suggesting that the use of the RDFixer ${ }^{\mathrm{TM}}$ software did not negatively impact any of the imaging outputs, such as image delays and/or mismatches between instrument movement and image. In addition, most surgeons perceived a wider field of view and better depth perception when the image was effectively corrected. When analyzing Table 1 , it is apparent that some surgeons did not perceive an image improvement with RD correction. However, this response was not consistent throughout all questions on the subjective survey. This lack of consistency might be due to the surgeons' low previous experience and therefore low comparability capacity. In further studies, a population of experienced $v s$ inexperienced arthroscopic surgeons should be enrolled to understand this variable.

Table 1. Correlation of demographic data with surgeons' answers to the subjective survey

\begin{tabular}{|c|c|c|c|c|c|c|c|c|c|c|c|}
\hline \multirow[b]{3}{*}{ Surgeon } & \multirow[b]{3}{*}{ Gender } & \multicolumn{4}{|c|}{ Demographic data } & \multicolumn{6}{|c|}{ Surgeons' answers to Subjective Survey } \\
\hline & & \multirow[b]{2}{*}{ Age } & \multirow[b]{2}{*}{$\begin{array}{l}\text { Year of } \\
\text { residency }\end{array}$} & \multirow[b]{2}{*}{$\begin{array}{c}\text { Hand } \\
\text { dominance }\end{array}$} & \multirow[b]{2}{*}{$\begin{array}{l}\text { Level of } \\
\text { expertise* }^{*}\end{array}$} & \multicolumn{2}{|c|}{$\begin{array}{c}\text { Perception of image } \\
\text { manipulation }\end{array}$} & \multicolumn{2}{|c|}{$\begin{array}{l}\text { Wider } \\
\text { field of view }\end{array}$} & \multicolumn{2}{|c|}{$\begin{array}{c}\text { Better } \\
\text { depth perception }\end{array}$} \\
\hline & & & & & & $\begin{array}{l}\text { Original } \\
\text { view }\end{array}$ & RDFixer & $\begin{array}{l}\text { Original } \\
\text { view }\end{array}$ & RDFixer & Original view & RDFixer \\
\hline 1 & $\mathrm{M}$ & 30 & $2^{\text {nd }}$ & Right & $<5$ & No & Yes & No & Yes & No & No \\
\hline 2 & $\mathrm{M}$ & 27 & $2^{\text {nd }}$ & Right & $<5$ & No & No & No & Yes & Yes & No \\
\hline 3 & $\mathrm{M}$ & 25 & $1^{\text {st }}$ & Right & $<5$ & No & No & Yes & Yes & No & Yes \\
\hline 4 & $\mathrm{M}$ & 32 & $2^{\text {nd }}$ & Right & $<5$ & Yes & Yes & Yes & Yes & No & Yes \\
\hline 5 & $\mathrm{M}$ & 28 & $3^{r d}$ & Right & [5-20] & Yes & No & No & Yes & No & No \\
\hline 6 & $\mathrm{M}$ & 31 & $2^{\text {nd }}$ & Right & $<5$ & No & No & No & No & No & Yes \\
\hline 7 & $\mathrm{~F}$ & 28 & $3^{r d}$ & Right & $<5$ & No & Yes & No & Yes & No & Yes \\
\hline 8 & $\mathrm{~F}$ & 29 & $5^{\text {th }}$ & Right & [5-20] & No & Yes & No & No & No & Yes \\
\hline 9 & $\mathrm{M}$ & 30 & $4^{\text {th }}$ & Right & $<5$ & No & Yes & No & Yes & No & Yes \\
\hline 10 & $\mathrm{M}$ & 34 & $6^{\text {th }}$ & Right & $<5$ & No & No & No & No & No & Yes \\
\hline 11 & $\mathrm{M}$ & 31 & $2^{\text {nd }}$ & Right & $<5$ & Yes & Yes & No & No & Yes & Yes \\
\hline 12 & $\mathrm{M}$ & 28 & $4^{\text {th }}$ & Right & $<5$ & No & No & No & No & No & No \\
\hline
\end{tabular}

${ }^{*}$ Number of previous arthroscopies. 


\section{Conclusion and future work}

The data generated in this pilot study confirmed the hypothesis that RDFixer ${ }^{\mathrm{TM}}$ provides an improved visualization experience that a surgeon can notice clearly, which influences surgical performance and the learning curve. This pilot study was designed to be performed by orthopedic surgeons who work with small-sized lenses with a wide field-of-view where the distortion effect is particularly strong. It is reasonable to assume that the observed improvements generalize to other surgical fields that use similar optics such as urology, neurosurgery and pediatric surgery.

Despite the positive trend, we recognize the need to expand the surgical assessment to other procedures within orthopedics and to other surgical fields. A broader population in both number and experience is also desirable, as are more quantitative metrics (such as execution time and number of surgical movements), to correlate the data with shorter learning curves and improved technical skills, which are of major interest to the surgical community.

\section{Conflict of interest}

The authors have stated explicitly that there are no conflicts of interest in connection with this article.

\section{Funding}

No specific funding.

\section{References}

1. Moreira-Pinto J, Lima E, Correia-Pinto J, Rolanda C. Natural orifice transluminal endoscopy surgery: a review. World $J$ Gastroenterol 2011; 17(33): 3795-3801.

2. Rolanda C, Lima E, Pêgo JM, et al. Third-generation cholecystectomy by natural orifices: transgastric and transvesical combined approach (with video). Gastrointest Endosc 2007; 65(1): 111-117.

3. Lima E, Rolanda C, Pêgo JM, et al. Transvesical endoscopic peritoneoscopy: a novel $5 \mathrm{~mm}$ port for intra-abdominal scarless surgery. J Urol 2006; 176(2): 802-805.

4. Fuchs KH. Minimally invasive surgery. Endoscopy 2002; 34(2): 154-159.

5. Harrell AG, Heniford T. Minimally invasive abdominal surgery: lux et veritas past, present, and future. Am J Surg 2005; 190: 239-243.

6. Tarin T, Feifer A, Kimm S, et al. Impact of a common clinical pathway on length of hospital stay in patients undergoing open and minimally invasive kidney surgery. $J$ Urol 2014; 191(5): $1225-1230$.
7. Dangelmajer S, Zadnik PL, Rodriguez ST, et al. Minimally invasive spine surgery for adult degenerative lumbar scoliosis. Neurosurg Focus 2014; 36(5): E7.

8. Breedveld P, Wentink M. Eye-hand coordination in laparoscopy an overview of experiments and supporting Aids. Minim Invasive Ther Allied Technol 2001; 10(3): 155-162.

9. Vakil N, Smith W, Bourgeois K, et al. Endoscopic measurement of lesion size: improved accuracy with image processing. Gastrointest Endosc 1994; 40(2 Pt 1): 178-183.

10. Way LW, Stewart L, Gantert W, et al. Causes and prevention of laparoscopic bile duct injuries: analysis of 252 cases from a human factors and cognitive psychology perspective. Ann Surg 2003; 237: 460-469.

11. Shin RB. Evaluation of the learning curve for laparoscopic Rouxen-Y gastric bypass surgery. Surg Obes Relat Dis 2005; 1: 91-94.

12. Suleman R, Yang T, Paige J, et al. Hand-eye dominance and depth perception effects in performance on a basic laparoscopic skills set. JSLS 2010; 14(1): 35-40.

13. Kirschniak A, Kunert W, Buess GF, Storz P. 3D HD versus 2D HD: surgical task efficiency in standardised phantom tasks. Surg Endosc 2012; 26: 1454-1460.

14. Manes R, Barnett S, Batra P. Utility of novel 3-dimensional stereoscopic vision system during endoscopic sinonasal and skull base surgery. Int Forum Allergy Rhinol 2011; 1(3): 191-197.

15. Taffinder N, Smith SG, Huber J, et al. The effect of a secondgeneration 3D endoscope on the laparoscopic precision of novices and experienced surgeons. Surg Endosc 1999; 13: 1087-1092.

16. Dion YM, Gaillard F. Visual integration of data and basic motor skills under laparoscopy. Influence of 2-D and 3-D video-camera systems. Surg Endosc 1997; 11: 995-1000.

17. Van Bergen P, Kunert W, Bessell J, Buess GF. Comparative study of two-dimensional and three-dimensional vision systems for minimally invasive surgery. Surg Endosc 1998; 12: 948-954.

18. Kari E, Oyesiku NM, Dadashev V, Wise SK. Comparison of traditional 2-dimensional endoscopic pituitary surgery with new 3dimensional endoscopic technology: intraoperative and early postoperative factors. Int Forum Allergy Rhinol 2012; 2: 2-8.

19. Liu K-C, Nicolau S, Wu H-S, et al. Enhanced minimally invasive surgery by 2D to 3D conversion. SID Symp Dig Tech Pap 2011; 42(1): $412-415$.

20. Pietrabissa A, Scarcello E, Carobbi A, Mosca F. Three-dimensional versus two-dimensional video system for the trained endoscopic surgeon and the beginner. Endosc Surg Allied Technol 1994; 2: 315-317.

21. Crosthwaite G, Chung T, Dunkley P, et al. Comparison of direct vision and electronic two- and three-dimensional display systems on surgical task efficiency in endoscopic surgery. Br J Surg 1995; 82(6): 849-851.

22. Hanna GB, Shimi SM, Cuschieri A. Randomised study of influence of two-dimensional versus three-dimensional imaging on performance of laparoscopic cholecystectomy. Lancet 1998; 51: 248-251.

23. Tabaee A, Anand VK, Fraser JF, et al. Three-dimensional endoscopic pituitary surgery. Neurosurgery 2009; 64: 288-293.

24. Helferty J, Zhang C, McLennan G, Higgins W. Videoendoscopic distortion correction and its application to virtual guidance of endoscopy. IEEE Trans Med Imag 2001; 20: 605-617.

25. Vakil N, Smith W, Bourgeois K, Everbach E, Knyrim K. Endoscopic measurement of lesion size: improved accuracy with image processing. Gastrointest Endosc 1994; 40: 178-183.

26. Pockett L, Salmimaa M, Pölönen M, Häkkinen J. The impact of barrel distortion on perception of stereoscopic scenes. SID Symp Dig Tech Pap 2010; 41(1): 526-529.

27. Melo R, Barreto JP, Falcão G. A new solution for camera calibration and real-time image distortion correction in medical endoscopy-initial technical evaluation. IEEE Trans Biomed Eng 2012; 59(3): 634-644.

28. Insel A, Carofino B, Leger R, et al. The development of an objective model to assess arthroscopic performance. J Bone Joint Surg Am 2009; 91(9): 2287-2295. 